\title{
A Systematic Review
}

\author{
Enrique Calvo-Ayala, MD; Babar A. Khan, MD; Mark O. Farber, MD, FCCP; \\ E. Wesley Ely, MD, MPH, FCCP; and Malaz A. Boustani, MD, MPH
}

\begin{abstract}
Background: ICU admissions are ever increasing across the United States. Following critical illness, physical functioning (PF) may be impaired for up to 5 years. We performed a systematic review of randomized controlled trials evaluating the efficacy of interventions targeting PF among ICU survivors. The objective of this study was to identify effective interventions that improve longterm PF in ICU survivors.

Methods: MEDLINE, Excerpta Medica Database (EMBASE), Cumulative Index to Nursing and Allied Health Literature (CINAHL), and Physiotherapy Evidence-Based Database (PEDro) were searched between 1990 and 2012. Two reviewers independently evaluated studies for eligibility, critically appraised the included studies, and extracted data into standardized evidence tables.

Results: Fourteen studies met the inclusion criteria. Interventions included exercise/physical therapy (PT), parenteral nutrition, nurse-led follow-up, spontaneous awakening trials, absence of sedation during mechanical ventilation, and early tracheotomy. Nine studies failed to demonstrate efficacy on PF of the ICU survivors. However, early physical exercise and PT-based interventions had a positive effect on long-term $P F$.

Conclusions: The only effective intervention to improve long-term PF in critically ill patients is exercise/PT; its benefit may be greater if started earlier. Further research in this area comparing different interventions and timing is needed. CHEST 2013; 144(5):1469-1480
\end{abstract}

Abbreviations: 6MWD = 6-min walk distance; ADL = activities of daily living; PEDro $=$ Physiotherapy Evidence-Based Database; $\mathrm{PF}=$ physical function; $\mathrm{PT}=$ physical therapy; $\mathrm{RCT}=$ randomized controlled trial; SF-36 PF $=$ Short Form-36 Physical Function questionnaire; $\mathrm{SR}=$ systematic review; TUG $=$ Timed Up and Go

$\mathbf{M}$ ore than 4 million ICU admissions occur annually in the United States. ${ }^{1}$ ICU beds account for $10 \%$ of all hospital beds and result in at least $20 \%$ of hospital operating costs amounting to $\$ 90$ billion annually. ${ }^{2}$ Since our population is progressively aging, and most ICU patients are $>65$ years of age, ICU care is expected to keep expanding in the next 2 decades. ${ }^{3,4}$ This increase in the numbers of critically ill patients, along with improvement in ICU mortality, is creating a growing number of ICU survivors. ${ }^{5}$

ICU survivors suffer from a variety of serious sequelae following their ICU stay, including late mortality, ongoing morbidity, neurocognitive defects, impaired mental health, poor functional capabilities, decreased quality of life, and decreased return to work and usual activities. ${ }^{6}$ Up to $69 \%$ of ICU survivors have clinically important long-term physical function $(\mathrm{PF})$ impair- ments. ${ }^{7-9}$ Immobility, depression, and cognitive and neuromuscular impairments have been postulated as risk factors for long-term PF impairment in survivors of critical illness. ${ }^{10}$ Post-ICU PF impairments are a major burden for patients, their families, and the health-care system because of high rates of institutionalization, frequent repeat hospitalizations, and other increased health-care services use. ${ }^{10}$

There has been enhanced interest in studying the etiology, pathophysiology, prevention, and treatment of PF impairment of ICU survivors, ${ }^{11}$ punctuated by the publication of three systematic reviews (SRs) in this field. ${ }^{12-14}$ Two of these reviews focused on describing the frequency, clinical features, and short-term outcomes (mortality, duration of mechanical ventilation, and length of hospitalization) related to the occurrence of neuromuscular abnormalities related 
to critical illness, without a particular focus on treatment strategies. ${ }^{12,14}$ The third SR aimed to assess the effect of any intervention, studied in a randomized controlled trial (RCT), on the incidence of critical illness polyneuropathy, critical illness myopathy, or both, which were diagnosed before hospital discharge. ${ }^{13}$ However, a review of effective therapies that may improve $\mathrm{PF}$ on or after hospital discharge in ICU survivors and the best timing to implement them, to our knowledge, has not been performed. We, therefore, performed a comprehensive, up-to-date SR looking for RCTs performed in critically ill subjects in which long-term PF (defined as PF assessed at the time of hospital discharge or later) was assessed as an outcome. Our primary goal was to identify therapies that were effective in improving $\mathrm{PF}$ while assessing the relationship between the timing of these therapies and the improvement in $\mathrm{PF}$.

\section{Materials AND Methods}

\section{Search Strategy}

We used a combination of controlled vocabulary and free text terms to search the following databases: MEDLINE, Excerpta Medica Database (EMBASE), Cumulative Index to Nursing and Allied Health Literature (CINAHL), and Physiotherapy EvidenceBased Database (PEDro) (e-Appendix 1). We filtered our search for treatment studies ${ }^{15-17}$ and all review articles, and cross-referenced studies from retrieved articles were screened for pertinent information (Fig 1). We restricted the search to articles published in the English language between January 1990 and December 2012.

\section{Study Selection}

Inclusion Criteria: RCTs were included if they met each of the following criteria: (1) Subjects were adults aged $\geq 18$ years admitted at some point to an ICU; (2) a treatment that can be

Manuscript received April 1, 2013; revision accepted July 14, 2013. Affiliations: From the Division of Pulmonary, Allergy, Critical Care, Occupational, and Sleep Medicine (Drs Calvo-Ayala, Khan, and Farber), and the Division of General Internal Medicine and Geriatrics (Dr Boustani), Department of Medicine, Indiana University School of Medicine, Indianapolis, IN; the Indiana University Center for Aging Research (Drs Khan and Boustani), Indianapolis, IN; the Regenstrief Institute (Drs Khan and Boustani), Indianapolis, IN; the Division of Allergy, Pulmonary, and Critical Care Medicine (Dr Ely), Department of Medicine, and the Center for Health Services Research (Dr Ely), Vanderbilt University School of Medicine, Nashville, TN; and the Veterans Affairs Tennessee Valley Geriatric Research, Education, and Clinical Center (Dr Ely), Nashville, TN.

A portion of these data were presented at the 2012 American Thoracic Society International Meeting, on May 18-23, 2012, San Francisco, CA.

Funding/Support: This study was supported by grants from the National Institute on Aging [Grant K23-AG043476 to Dr Khan and Grant R01AG034205 to Dr Boustani].

Correspondence to: Babar A. Khan, MD, 410 W 10th St, Ste 2000, Indianapolis, IN 46202; e-mail: bakhan@iupui.edu (C) 2013 American College of Chest Physicians. Reproduction of this article is prohibited without written permission from the American College of Chest Physicians. See online for more details. DOI: 10.1378/chest.13-0779 applied to any patient in the ICU was compared with placebo, no treatment, or a different treatment; and (3) the primary or secondary outcomes were long-term measures of $\mathrm{PF}$ or its surrogates that occurred after the intervention was applied (a baseline functional assessment alone would not meet the study eligibility). Acceptable measures of PF included but were not limited to muscle strength testing, functional tests and walk tests, and patientcentered outcomes, such as health-related quality of life. ${ }^{11}$ For purposes of this review, an outcome was defined as long-term if it occurred on or after hospital discharge.

Exclusion Criteria: Studies were excluded (1) if they enrolled patients $<18$ years of age; $(2)$ if they were done primarily in patients with acquired brain injury (defined as head trauma, hypoxia, infection, tumor, substance abuse, degenerative neurologic disease, stroke, intracranial bleeding) or underlying neuropathies/myopathies; (3) if the reported outcome was measured before the day of hospital discharge or the specific time of measurement in relation to hospital discharge was not mentioned; (4) if the intervention was not generalizable to all patients in the ICU (eg, cardiac revascularization); or (5) if the studied population was recruited from long-term acute-care hospitals.

\section{Data Abstraction and Quality Assessment}

Titles and abstracts were screened by two reviewers (E. C.-A., B. A. K.). Full text of potentially relevant studies was assessed. Original authors were contacted for additional information if a long-term outcome measurement was described in the Methods section of the article but its results were not available in the Results portion or if they were reported in a way that treatment groups were not compared. The reviewers independently assessed and abstracted pertinent data from trials in duplicate using a standardized, predefined form. Abstracted data included each study's methodology, setting, baseline patient characteristics, intervention, outcomes, and follow-up. The methodological quality of each trial was assessed using the PEDro scale (e-Appendix 2). This is an 11-item tool, developed to measure the methodological quality of randomized and quasi-randomized controlled trials of physiotherapy interventions, deriving a score of 0 to 10 , with higher scores indicating higher quality. The PEDro scale was used given the nature of some therapies that were included (where double-blinding is often not possible because of their nature), because it breaks down the levels of blinding and accounts for concealed allocation. ${ }^{18-21}$ The risk of bias was intrinsically evaluated with the methodological assessment, because the PEDro score includes the domains recommended by the Cochrane Handbook for this purpose. ${ }^{22}$

Any disagreements related to either inclusion or exclusion criteria and quality assessment were resolved by discussions and consensus between the reviewers. The studies included in this review were heterogeneous in regard to their interventions, control intervention, clinical setting, and population, and therefore were not amenable to be merged into a pooled meta-analytic summary. Hence, we were unable to perform a funnel plot to detect publication bias.

\section{RESULTS}

The original search strategy identified 319,957 potential eligible studies (Fig 1). After application of study terms, the majority were excluded. A manual detailed review of the remaining 10,138 abstracts and full-text versions revealed 14 trials that met all inclusion criteria and were considered appropriate for inclusion in our SR. 


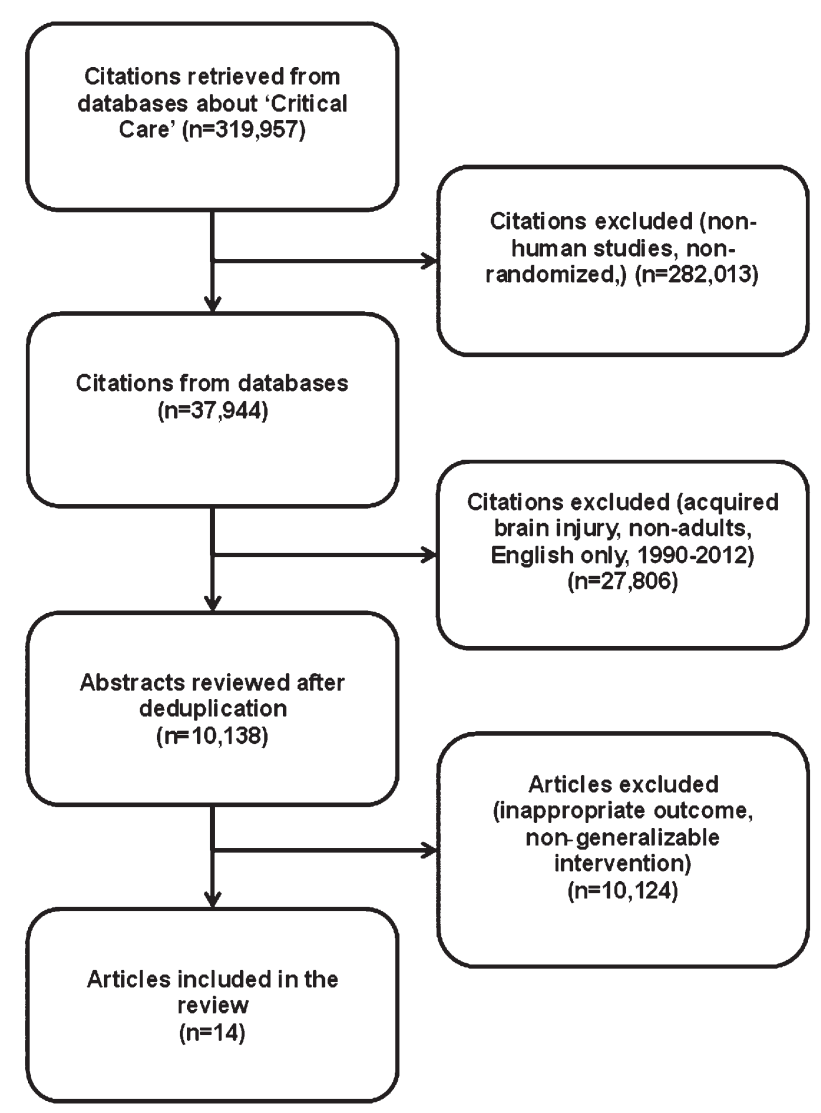

FIGURE 1. Search results.

Tables 1 and 2 show the characteristics of the 14 included studies. ${ }^{23-36}$ Trials were conducted in six countries (Australia, Belgium, Denmark, France, United Kingdom, and United States). There were five multicenter studies (range, 2-12 centers per study).24,25,28,32,34 Study sizes ranged widely (16-4,640 subjects), with only three trials enrolling $>300$ patients. ${ }^{24,27,29}$ The study participants encompass a broad distribution of adult ICU patients evidenced by the variations in the mean APACHE (Acute Physiology and Chronic Health Evaluation) II score (range, 9-28) and the mean age of studies' subjects (48-66 years).

Described interventions were exercise/physical therapy (PT), ${ }^{23,26,28,30,32-34}$ parenteral nutrition, ${ }^{24}$ nurseled follow-up, ${ }^{25,27}$ intensive insulin therapy, ${ }^{29}$ spontaneous awakening and breathing trials, ${ }^{31}$ absence of sedation during mechanical ventilation, ${ }^{35}$ and early tracheotomy ${ }^{36} \mathrm{PF}$ was evaluated most commonly through the Short Form-36 PF questionnaire (SF-36 PF) (eight studies). ${ }^{23,25,26,28,30,32,35,36}$ Other PF outcomes included the Barthel Index, ${ }^{34}$ the 6-min walk distance (6MWD), 23,24,26,30,31,34 and the ability to perform activities of daily living (ADL). ${ }^{24,30,31,34}$

None of the studies reached the maximum PEDro score of 10 for quality assessment. This was most commonly due to the lack of double-blinding associated with the nature of the studied interventions (eg, exer- cise, physical therapies, self-help manual, and telephone follow-up). In five trials, the baseline characteristics of the population were dissimilar among the studied groups. $23,27,30,33,36,37$ In only two of the studies were the study subjects blinded to the intervention. ${ }^{29,32}$ Five of the studies failed to report at least one measured key outcome (not necessarily PF outcome) in at least $85 \%$ of the allocated subjects, ${ }^{23,25,29,31,35}$ and only four of the trials reported a $\mathrm{PF}$ outcome in $>80 \%$ of the recruited subjects. ${ }^{28,30,32,34} \mathrm{PF}$ or its surrogate was not the primary outcome measured in six of the trials (as defined as the outcome used for sample size calculation or, if this was not reported, defined as the outcome described in the manuscript as primary outcome). 24,29,31,32,35,36

Only five of the 14 included studies reported positive effects on PF outcomes (Fig 2).23,26,30,32,34 All the positive trials studied exercise/PT protocols in the treatment arms. The first study that provided positive results was published in 2003 by Jones et al. ${ }^{32}$ This multicenter trial tested the effectiveness of a 93-page self-help rehabilitation manual provided after ICU discharge to subjects who were on mechanical ventilation for $>48 \mathrm{~h}$; this intervention was associated with a statistically significant improvement of the SF-36 $\mathrm{PF}$ scores at 8 weeks and 6 months after hospital discharge compared with control subjects $(P=.006)$. Two positive trials were published in 2009..23,34 Burtin et al ${ }^{23}$ performed a trial in critically ill subjects from a university hospital with an expected prolonged ICU course using a daily exercise training session during the ICU stay as intervention compared with usual care. The intervention group had better SF-36 PF scores $(P<.01)$ and was able to walk more distance in the 6MWD $(P<.05)$, but there was no statistically significant difference in handgrip force or Berg Dyspnea Scale. The study by Schweickert et $\mathrm{al}^{34}$ was performed in two university hospitals and included sedated adults with baseline functional independence who had been on mechanical ventilation for $<72 \mathrm{~h}$ and were randomly assigned to early exercise and mobilization during periods of daily interruption of sedation vs usual care. At hospital discharge, the proportion of subjects who were able to walk and perform six ADL was higher in the intervention group $(P=.02)$; also, the Barthel Index Score and the walking distance outcomes were better in the intervention group $(P=.05$ and $P=.004$, respectively). Denehy et a ${ }^{26}$ disclosed preliminary data in 2011 of a single-center trial of subjects admitted to an ICU for $\geq 5$ days, in which an "intensive rehabilitation protocol" was compared with usual care. Intervention subjects were able to cover a longer distance in the 6MWD test, which was statistically significant after correcting for time effect. There were no statistically significant differences in quality-of-life scores (SF-36) or the Timed Up and Go (TUG) test. The most recent 


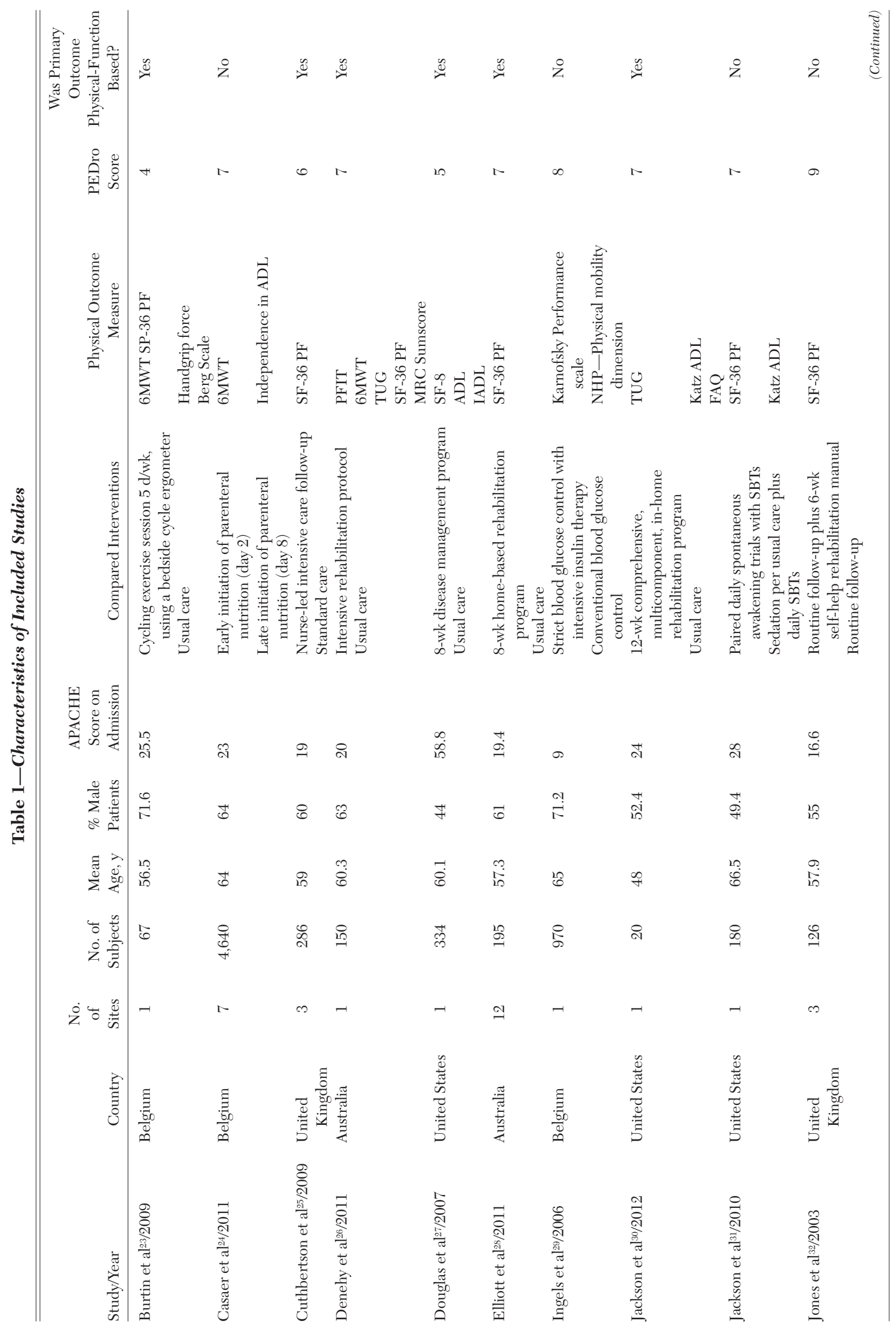




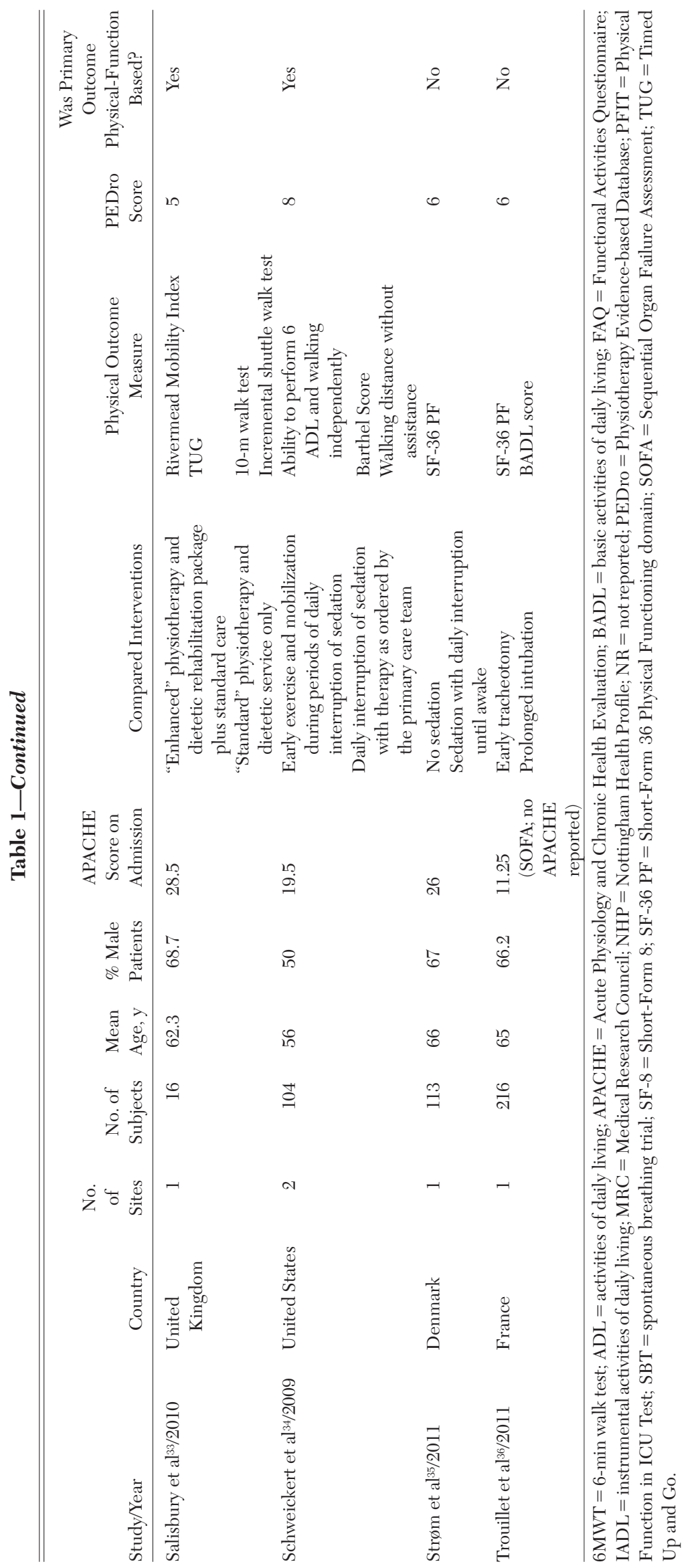




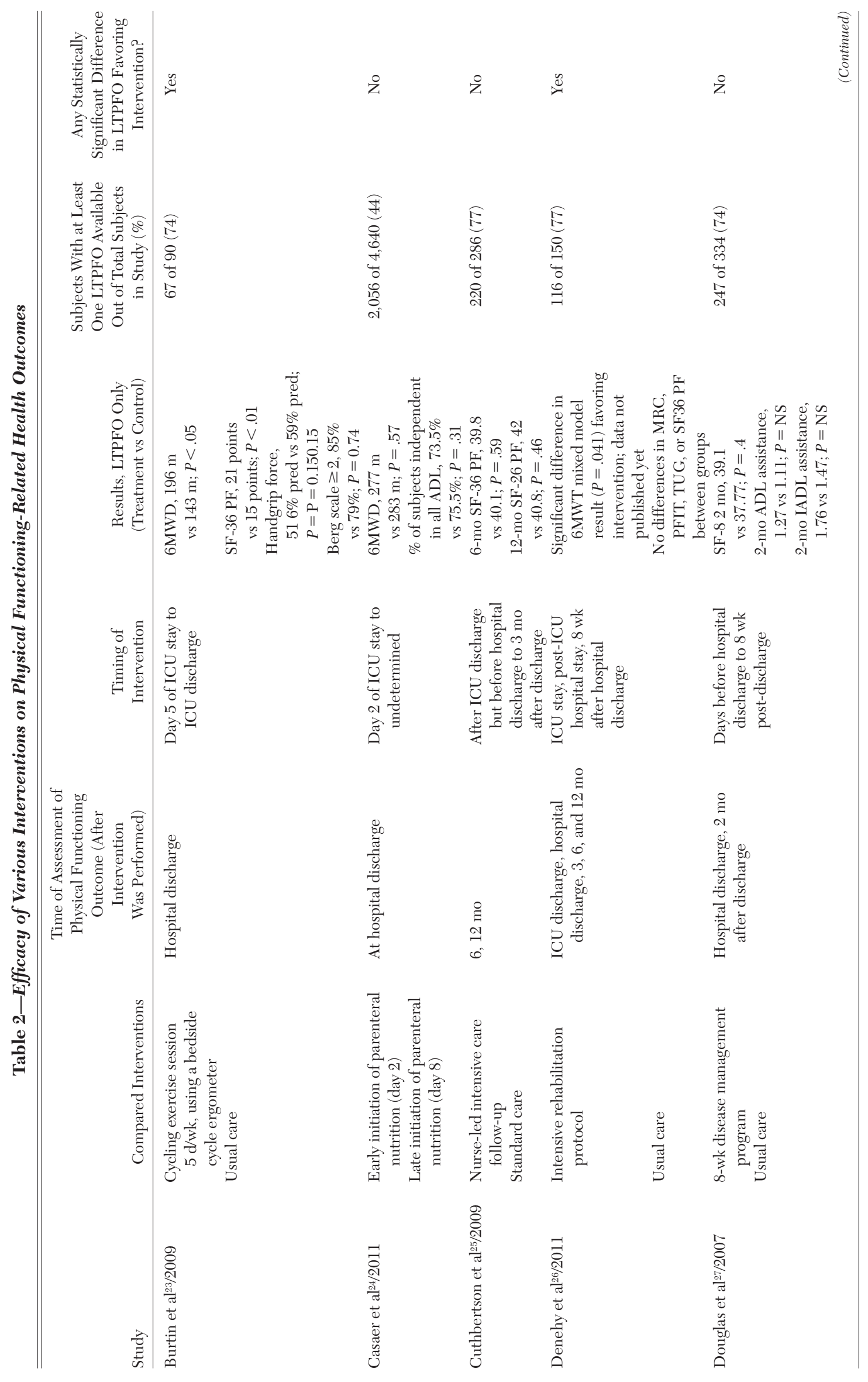




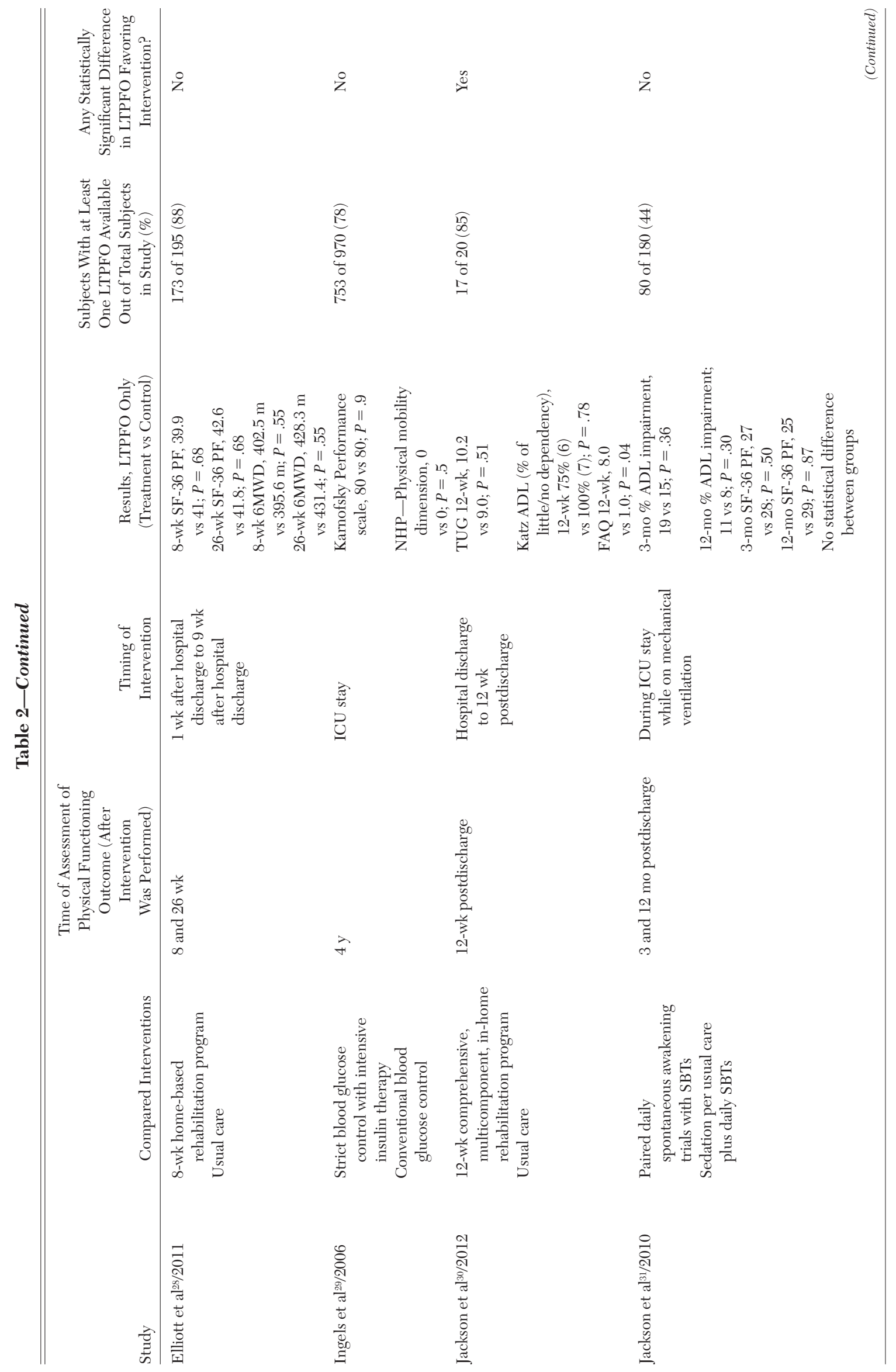




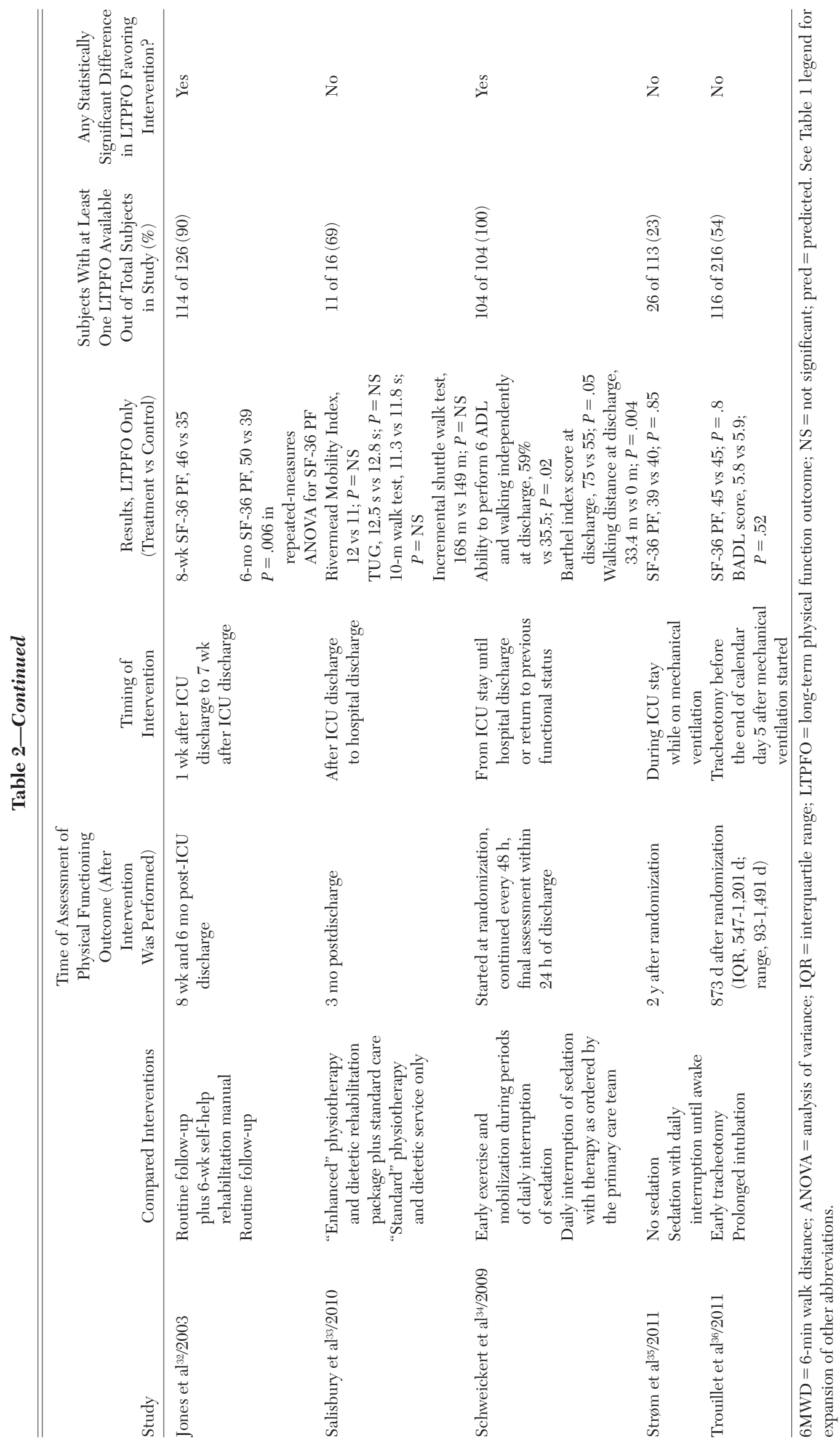




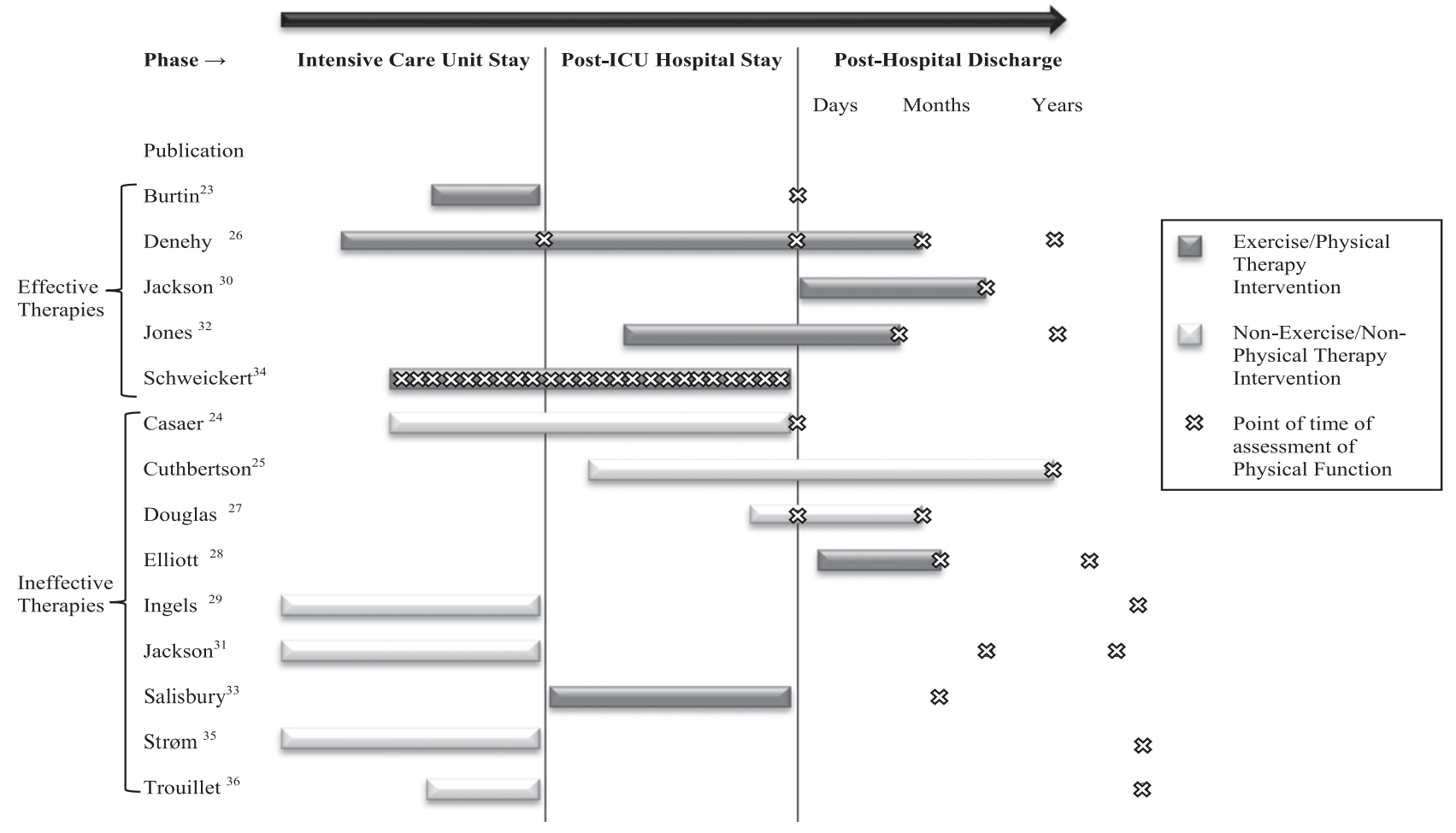

FIGURE 2. Timing of interventions and physical function assessment of the included trials.

positive report was published in 2012 by Jackson et $\mathrm{al}^{30}$; this was a single-center trial that included adult subjects who had an ICU length of stay $>5$ days and were recruited after ICU discharge. The examined intervention was a multicomponent rehabilitation program that was started after hospital discharge. A statistically significant difference favoring the intervention in the Functional Assessment Questionnaire was found; however, there were no differences between groups in ADL or TUG scores.

There were two reports of trials that used exercise/PT as the studied intervention that failed to demonstrate a positive treatment effect. Salisbury et al ${ }^{33}$ did a small trial of 16 patients comparing an "enhanced rehabilitation" (which consisted of interventions considered additional to usual care, such as "supervised passive, active and strengthening exercises, facilitation of additional transfers and mobility practice, balance exercises and advice") with usual care. No significant differences were found between the studied groups for any of the PF outcomes (Rivermead Mobility Index, TUG test, 10-min walk test, and incremental shuttle walk test). The other study that failed to show an advantage of exercise/PT as an intervention was performed by Elliot et al, ${ }^{28}$ who randomized adults admitted to the ICU for at least $48 \mathrm{~h}$ and mechanically ventilated for $\geq 24 \mathrm{~h}$ to receive usual care vs an individualized endurance and strength training program for 8 weeks after hospital discharge. After 8 weeks of intervention, no significant differences were found in 6MWD or SF-36 scores between the studied groups.

None of the trials studying medications or devices showed better outcomes for long-term PF. Timing of interventions and their relationship with the $\mathrm{PF}$ assessment can be seen in Figure 2. Interventions were performed (1) only during ICU stay in five studies, $23,29,31,35,36$ (2) started in the ICU but continued after ICU stay in three studies, ${ }^{24,26,34}$ and (3) started after ICU discharge in the remaining six studies. ${ }^{25,27,28,30,32,33}$ Four of the five trials with positive findings described interventions that were started before hospital discharge (one performed the intervention during ICU stay only, ${ }^{23}$ two started treatment in the ICU that was continued after ICU discharge, ${ }^{26,34}$ and one started the protocol after ICU stay but before hospital discharge). ${ }^{32}$ A dose-dependent effect for effective interventions could not be estimated, given that only three reports of exercise/PT interventions described the frequency and/or duration of treatment. ${ }^{23,26,34}$ In three of the studies with positive results, frequency and duration of the therapy was variable, as it was either individualized after assessment by a therapist ${ }^{30,34}$ or it relied on the compliance of each subject with a manual. ${ }^{32}$

\section{Discussion}

Our SR found that the only effective intervention in improving long-term $\mathrm{PF}$ is exercise/PT. Based on 
the reviewed studies, it seems that if the intervention is started earlier, better outcomes may be obtained, although no trials comparing early vs late PT intervention directly were identified. We were unable to establish a dose-dependent effect, as most of the reports had individualized programs that varied from subject to subject, with different intensities and frequencies within each study. Other studied therapies (tight glucose control, early parenteral nutrition, tracheotomy, disease management program) failed to show a statistically significant effect. Our findings are in agreement with previous narrative reviews, ${ }^{38,39}$ expert opinions, ${ }^{40,41}$ and clinical practice guidelines. ${ }^{42}$

The quality of the included trials was not the best for variety of reasons. First, the intrinsic characteristics of the studied interventions make blinding to subjects and investigators difficult. Also, there was a high rate of drop-outs, which may reflect the technical difficulty of performing clinical trials in critical care survivors due to known issues of this population (eg, high mortality rates, loss to follow-up) and a survivor bias (patients who died before the assessment may have had worse physical impairment than those who survived and were assessed).

The strengths of our review include a comprehensive and very sensitive search strategy (avoiding the exclusion of potential studies), the inclusion of the best available evidence by including only RCTs, and attempts to identify unpublished data. However, our review has limitations. Although our literature search procedures were extensive, other trials may have appeared in non-English-language publications or may have not been published. Publication bias may occur, resulting in an overestimation of the efficacy of these treatments. As for the research itself, many studies investigating critical care survivors have usually measured short-term PF outcomes, but only few have evaluated long-term PF outcomes. Given the heterogeneity of the included studies (size, interventions, and outcomes), and the quantity and quality of the available evidence, drawing solid conclusions is difficult. This is probably intrinsic to our SR design, because we were looking at "any" intervention that reported certain outcomes (which is opposite to most SRs, which look for certain interventions that report certain outcomes). Also, our definition of long-term follow-up (at hospital discharge or later) is arbitrary and probably does not reflect the actual needs of longterm follow-up for these patients, which still need to be established. Because of this definition, we may have included trials that may not be clinically relevant to reallife long-term follow-up. Six trials reported outcomes at $\geq 6$ months after hospital discharge, ${ }^{25,26,28,29,32,36}$ and from these only two were positive..$^{26,32}$
It is difficult to predict how easily the presented results will be applied and implemented into regular clinical practice. Although the topic of long-term poor outcome after critical care has been a subject of discussion in the last decade, ${ }^{6,43,44}$ the implementation of strategies to reduce physical dysfunction after critical illness seems challenging. For example, to implement some of the effective inpatient interventions, an assumption that there are dedicated personnel for PT in the critical care setting all days must be made. Previous reports have documented the variability in the availability of therapists on a daily basis in ICUs ${ }^{45}$ and the low frequency of PT occurrence in critically ill patients. ${ }^{38}$ The cost-effectiveness of having a dedicated therapist in the ICU still needs further assessment for these therapies to be implemented successfully in routine clinical settings. It is well known that interventions that have shown positive outcomes in studies of ICU subjects (eg, daily interruption of sedation), often have not been implemented in the community after years of proven benefit ${ }^{46}$; thus, the application of a treatment requiring a dedicated therapist, although important, seems difficult to implement in regular clinical practice. Similarly, interventions like the one described by Jackson et al, ${ }^{30}$ which require remote follow-up of patients at their homes using teletechnology and a trained social worker, are difficult to implement outside of a research setting.

We identified gaps and biases in available evidence that suggest directions for future research. First, the fact that only two pharmaceutical interventions have been assessed for their effect on long-term PF of critical care survivors is striking. Both failed to show an advantage in chronic PF outcomes. ${ }^{24,29}$ However, these studies were not powered to show an improvement specifically in the PF outcome. The case of the tracheotomy intervention is similar (not effective, yet not powered to demonstrate an advantage in $\mathrm{PF}$ ). It would be interesting to see if critical care survivors enrolled in trials of other pharmaceutical interventions could be followed long-term for improved PF outcomes. Other interventions that have been tested for ICU-acquired weakness (eg, electrical muscle stimulation $)^{47}$ may have a role in preventing and treating long-term physical dysfunction in ICU survivors.

Another gap identified in the available evidence is the lack of consensus regarding the setting, timing, and duration of follow-up survivors of critical illness. This is easily demonstrated by the different instruments used to assess PF, the different points and length of time that the subjects were followed in every study, and the different interventions tested. Similarly to the case of ICU-acquired weakness, ${ }^{48}$ there is a need to define the best tools and timing to make trials and interventions comparable in the future. 
In conclusion, based on available evidence, early exercise/PT seems to be the only treatment yet shown to improve long-term PF of ICU survivors. The feasibility of implementation for these effective therapies in the community still needs to be proven. Efforts in the critical care community to standardize long-term follow-up in terms of tools, frequency, and length of time are required. New multicenter trials, testing head-to-head early vs late interventions, assessing patients for longer periods of time after discharge, and comparing these proven therapies with new ones are needed.

\section{ACKNOWLEDGMENTS}

Author contributions: Dr Khan acts as a guarantor of the manuscript.

Dr Calvo-Ayala: contributed to conception and design of the study; study selection, data extraction, quality assessment, data analysis, and/or interpretation; writing or revising the manuscript for important intellectual content; and approval of the final manuscript; and served as principal author.

Dr Khan: contributed to conception and design of the study; data acquisition, analysis, and/or interpretation; writing or revising the manuscript for important intellectual content; and approval of the final manuscript.

Dr Farber: contributed to data analysis and interpretation, writing or revising the manuscript for important intellectual content, and approval of the final manuscript.

Dr Ely: contributed to writing or revising the manuscript for important intellectual content and approval of the final manuscript.

Dr Boustani: contributed to conception and design of the study, data analysis, and/or interpretation, critical revision of the manuscript, and approval of the final manuscript.

Financial/nonfinancial disclosures: The authors have reported to CHEST that no potential conflicts of interest exist with any companies/organizations whose products or services may be discussed in this article.

Role of sponsors: The sponsor had no role in the design of the study, the collection and analysis of the data, or in the preparation of the manuscript.

Other contributions: We thank Kellie Kaneshiro, BA, AMLS, who helped in the development of the search strategies and undertook the searches.

Additional information: The e-Appendixes can be found in the "Supplemental Materials" area of the online article.

\section{REFERENCES}

1. Joint Commission Resources Inc. Improving Care in the ICU. Oakbrook Terrace, IL: Joint Commission Resources; 2004.

2. Pronovost PJ, Needham DM, Waters H, et al. Intensive care unit physician staffing: financial modeling of the Leapfrog standard. Crit Care Med. 2004;32(6):1247-1253.

3. Hall MJ, Owings MF. 2000 National Hospital Discharge Survey. Adv Data. 2002; (329):1-18.

4. Needham DM, Bronskill SE, Calinawan JR, Sibbald WJ, Pronovost PJ, Laupacis A. Projected incidence of mechanical ventilation in Ontario to 2026: preparing for the aging baby boomers. Crit Care Med. 2005;33(3):574-579.

5. Spragg RG, Bernard GR, Checkley W, et al. Beyond mortality: future clinical research in acute lung injury. Am J Respir Crit Care Med. 2010;181(10):1121-1127.

6. Angus DC, Carlet J; 2002 Brussels Roundtable Participants. Surviving intensive care: a report from the 2002 Brussels Roundtable. Intensive Care Med. 2003;29(3):368-377.
7. Angus DC, Clermont G, Linde-Zwirble WT, et al; NO-06 Investigators. Healthcare costs and long-term outcomes after acute respiratory distress syndrome: a phase III trial of inhaled nitric oxide. Crit Care Med. 2006;34(12):2883-2890.

8. van der Schaaf M, Beelen A, Dongelmans DA, Vroom MB, Nollet F. Functional status after intensive care: a challenge for rehabilitation professionals to improve outcome. J Rehabil Med. 2009;41(5):360-366.

9. van der Schaaf M, Beelen A, Dongelmans DA, Vroom MB, Nollet F. Poor functional recovery after a critical illness: a longitudinal study. J Rehabil Med. 2009;41(13):1041-1048.

10. Needham DM, Feldman DR, Kho ME. The functional costs of ICU survivorship. Collaborating to improve post-ICU disability. Am J Respir Crit Care Med. 2011;183(8):962-964.

11. Elliott D, Denehy L, Berney S, Alison JA. Assessing physical function and activity for survivors of a critical illness: a review of instruments. Aust Crit Care. 2011;24(3):155-166.

12. De Jonghe B, Cook D, Sharshar T, Lefaucheur JP, Carlet J, Outin H. Acquired neuromuscular disorders in critically ill patients: a systematic review. Groupe de Reflexion et d'Etude sur les Neuromyopathies En Reanimation. Intensive Care Med. 1998;24(12):1242-1250.

13. Hermans G, De Jonghe B, Bruyninckx F, Van den Berghe G. Interventions for preventing critical illness polyneuropathy and critical illness myopathy. Cochrane Database Syst Rev. 2009; (1):CD006832.

14. Stevens RD, Dowdy DW, Michaels RK, Mendez-Tellez PA, Pronovost PJ, Needham DM. Neuromuscular dysfunction acquired in critical illness: a systematic review. Intensive Care Med. 2007;33(11):1876-1891.

15. Wong SS, Wilczynski NL, Haynes RB. Developing optimal search strategies for detecting clinically sound treatment studies in EMBASE. J Med Libr Assoc. 2006;94(1):41-47.

16. McKibbon KA, Wilczynski NL, Haynes RB, Hedges T; Hedges Team. Retrieving randomized controlled trials from medline: a comparison of 38 published search filters. Health Info Libr J. 2009;26(3):187-202.

17. Wong SS, Wilczynski NL, Haynes RB. Optimal CINAHL search strategies for identifying therapy studies and review articles. J Nurs Scholarsh. 2006;38(2):194-199.

18. Macedo LG, Elkins MR, Maher CG, Moseley AM, Herbert RD, Sherrington C. There was evidence of convergent and construct validity of Physiotherapy Evidence Database quality scale for physiotherapy trials. J Clin Epidemiol. 2010;63(8): 920-925

19. Bhogal SK, Teasell RW, Foley NC, Speechley MR. The PEDro scale provides a more comprehensive measure of methodological quality than the Jadad scale in stroke rehabilitation literature. J Clin Epidemiol. 2005;58(7):668-673.

20. de Morton NA. The PEDro scale is a valid measure of the methodological quality of clinical trials: a demographic study. Aust J Physiother. 2009;55(2):129-133.

21. Elkins MR, Herbert RD, Moseley AM, Sherrington C, Maher C. Rating the quality of trials in systematic reviews of physical therapy interventions. Cardiopulm Phys Ther J. 2010;21(3):20-26.

22. Higgins JPT, Green S. Cochrane Collaboration. Cochrane Handbook for Systematic Reviews of Interventions. Chichester, England: Wiley-Blackwell; 2008.

23. Burtin C, Clerckx B, Robbeets C, et al. Early exercise in critically ill patients enhances short-term functional recovery. Crit Care Med. 2009;37(9):2499-2505.

24. Casaer MP, Mesotten D, Hermans G, et al. Early versus late parenteral nutrition in critically ill adults. $N$ Engl J Med. 2011; 365(6):506-517.

25. Cuthbertson BH, Rattray J, Campbell MK, et al. The PRaCTICaL study of nurse led, intensive care follow-up 
programmes for improving long term outcomes from critical illness: a pragmatic randomised controlled trial. BMJ. 2009; 339:b3723.

26. Denehy L, Berney S, Skinner E, et al. Evaluation of exercise rehabilitation for survivors of intensive care: an assessor blinded randomised controlled trial [abstract]. Am J Respir Crit Care Med. 2011;183:A2642.

27. Douglas SL, Daly BJ, Kelley CG, O’Toole E, Montenegro H. Chronically critically ill patients: health-related quality of life and resource use after a disease management intervention. Am J Crit Care. 2007;16(5):447-457.

28. Elliott D, McKinley S, Alison J, et al. Health-related quality of life and physical recovery after a critical illness: a multicentre randomised controlled trial of a home-based physical rehabilitation program. Crit Care. 2011;15(3):R142.

29. Ingels C, Debaveye Y, Milants I, et al. Strict blood glucose control with insulin during intensive care after cardiac surgery: impact on 4-years survival, dependency on medical care, and quality-of-life. Eur Heart J. 2006;27(22):2716-2724.

30. Jackson JC, Ely EW, Morey MC, et al. Cognitive and physical rehabilitation of intensive care unit survivors: results of the RETURN randomized controlled pilot investigation. Crit Care Med. 2012;40(4):1088-1097.

31. Jackson JC, Girard TD, Gordon SM, et al. Long-term cognitive and psychological outcomes in the awakening and breathing controlled trial. Am J Respir Crit Care Med. 2010;182(2):183-191.

32. Jones C, Skirrow P, Griffiths RD, et al. Rehabilitation after critical illness: a randomized, controlled trial. Crit Care Med. 2003;31(10):2456-2461.

33. Salisbury LG, Merriweather JL, Walsh TS. The development and feasibility of a ward-based physiotherapy and nutritional rehabilitation package for people experiencing critical illness. Clin Rehabil. 2010;24(6):489-500.

34. Schweickert WD, Pohlman MC, Pohlman AS, et al. Early physical and occupational therapy in mechanically ventilated, critically ill patients: a randomised controlled trial. Lancet. 2009;373(9678):1874-1882.

35. Strøm T, Stylsvig M, Toft P. Long-term psychological effects of a no-sedation protocol in critically ill patients. Crit Care. 2011;15(6):R293.

36. Trouillet JL, Luyt CE, Guiguet M, et al. Early percutaneous tracheotomy versus prolonged intubation of mechanically ventilated patients after cardiac surgery: a randomized trial. Ann Intern Med. 2011;154(6):373-383.

37. Salisbury LG, Merriweather JL, Walsh TS. Rehabilitation after critical illness: could a ward-based generic rehabilitation assistant promote recovery? Nurs Crit Care. 2010;15(2): $57-65$.

38. Truong AD, Fan E, Brower RG, Needham DM. Bench-tobedside review: mobilizing patients in the intensive care unit-from pathophysiology to clinical trials. Crit Care. 2009; $13(4): 216$.

39. Needham DM. Mobilizing patients in the intensive care unit: improving neuromuscular weakness and physical function. JAMA. 2008;300(14):1685-1690.

40. Hanekom S, Gosselink R, Dean E, et al. The development of a clinical management algorithm for early physical activity and mobilization of critically ill patients: synthesis of evidence and expert opinion and its translation into practice. Clin Rehabil. 2011;25(9):771-787.

41. Griffiths RD, Jones C. Seven lessons from 20 years of follow-up of intensive care unit survivors. Curr Opin Crit Care. 2007;13(5):508-513.

42. Centre for Clinical Practice. Rehabilitation After Critical Illness. NICE Clinical Guidelines, No. 83. London, England: National Institute for Health and Clinical Excellence; 2009.

43. Kaplan V, Angus DC. Surviving intensive care. Crit Care Med. 2002;30(3):703-705.

44. Desai SV, Law TJ, Needham DM. Long-term complications of critical care. Crit Care Med. 2011;39(2):371-379.

45. Hodgin KE, Nordon-Craft A, McFann KK, Mealer ML, Moss M. Physical therapy utilization in intensive care units: results from a national survey. Crit Care Med. 2009;37(2):561-566., quiz 566-568.

46. Miller MA, Bosk EA, Iwashyna TJ, Krein SL. Implementation challenges in the intensive care unit: the why, who, and how of daily interruption of sedation. J Crit Care. 2012;27(2):218. e1-7.

47. Routsi C, Gerovasili V, Vasileiadis I, et al. Electrical muscle stimulation prevents critical illness polyneuromyopathy: a randomized parallel intervention trial. Crit Care. 2010;14(2): R74.

48. Proceedings of a round table conference in Brussels, Belgium, March 2009. Crit Care Med. 2009;37(suppl 10):S295-S461. 